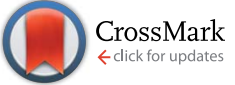

Cite this: RSC Adv., 2016, 6, 86269

\title{
Amino acid recognition by fine tuning the association constants: tailored naphthalimides in pillar[5]arene-based indicator displacement assays $\uparrow$
}

\begin{abstract}
Márton Bojtár, ${ }^{a}$ Adrien Paudics, ${ }^{\text {b }}$ Dóra Hessz, ${ }^{\text {bc }}$ Miklós Kubinyi ${ }^{\text {bc }}$ and István Bitter ${ }^{* a}$
Three aminonaphthalimide derivatives were synthesized bearing different anchoring groups in their 4-position in order to adjust the supramolecular interactions with carboxylato-pillar[5]arene (WP5), an anionic, water-soluble host. The modification of the anchor groups resulted in varying association constants embracing three orders of magnitude $\left(K_{\mathrm{a}}\right.$ from $\sim 10^{3}$ to $\sim 10^{6}$ ) in buffered water. Since the fluorophore responded significantly to the electronic environment, large fluorescence quenching was observed with the anionic WP5 host. The naphthalimide indicator-WP5 supramolecular assemblies were used to detect arginine and lysine with complete selectivity over other non-basic $\alpha$-amino acids by turnon fluorescence. The same assemblies proved to be highly sensitive fluorescence displacement assays for the detection of cadaverine.
\end{abstract}

Received 9th June 2016

Accepted 3rd September 2016

DOI: $10.1039 / c 6 r a 15003 a$

www.rsc.org/advances

usually ionic (carboxylato-, ${ }^{18,19}$ ammonium, ${ }^{20,21}$ imidazolium $^{22}$ and recently, phosphoryl ${ }^{23}$ ) groups are emerging as popular hosts in aqueous media. Carboxylato-pillar[ $n]$ arenes (WPns), first synthesized by Ogoshi in 2010 (ref. 18) $(n=5)$ are interesting structures due to their hydrophobic, electron-rich cavity and anionic outer rim. These macrocycles are ideal hosts for ammonium-containing and electron-deficient guests. ${ }^{18,24-27}$ As was demonstrated, WP5 forms strong complexes with basic amino acids and cadaverine, the interactions were, however, monitored only by NMR-studies. ${ }^{28}$

As a first example for a chemosensor, a pillar[5]arene functionalized with a pyrene fluorophore was synthesized by Stoddart to detect diamines in acetonitrile/water mixtures. ${ }^{29}$ This was followed by FID assays with WPn hosts. The operations of a quaternary ammonium attached to tetraphenylethene ${ }^{30}$ and an $N$-methylacridinium ${ }^{31}$ fluorescent guest in WP6 and WP5, respectively, were demonstrated as FID assays for paraquat. Recently, we reported the complexation of 4 -styryl- $N$-methylpyridinium iodide and two other stilbazolium dyes with WP5 in water ${ }^{32,33}$ and proposed a FID system for the colorimetric and fluorescent detection of paraquat with a detection limit of 0.2 $\mu \mathrm{M}$. To our knowledge, no pillar[5]arene-based indicator displacement assays were constructed for analytes with biological relevance, such as amino acids (AAs).

Detection and quantification of AAs and related compounds is mainly based on chromatographic ${ }^{34}$ and electrochemical ${ }^{35}$ methods. Besides, in the past years, there has been a rapid progress in the development of fluorescent and colorimetric chemosensors for the detection of AAs, ${ }^{36}$ however there is still a need to increase the selectivity and simplicity of these systems. Basic amino acids, arginine (Arg) and lysine (Lys) have $\mathrm{N}$-containing units on the side chain that is vital for the proper

${ }^{a}$ Department of Organic Chemistry and Technology, Budapest University of Technology and Economics, 1521 Budapest, Hungary. E-mail: bitter@oct.bme.hu

${ }^{b}$ Department of Physical Chemistry and Materials Science, Budapest University of Technology and Economics, 1521 Budapest, Hungary

'Institute of Materials and Environmental Chemistry, Research Center for Natural Sciences, Hungarian Academy of Sciences, 1519 Budapest, P. O. B. 286, Hungary

$\dagger$ Electronic supplementary information (ESI) available. See DOI: 10.1039/c6ra15003a 
function of the living systems. Their fluorescent and/or colorimetric detection is usually achieved by metal complexes, ${ }^{37-40}$ metal nanoparticles, ${ }^{41-43}$ cucurbituril- or cucurbituril-analoguebased differential sensing platforms ${ }^{4-46}$ or by utilizing their nucleophilicity and basicity for bond cleavage. ${ }^{47,48}$ Biogenic diamines are usually detected by non-selective amine chemosensors $^{49-55}$ or cucurbituril-based FID systems. ${ }^{4}$

In this paper, our aim has been to introduce a novel principle to construct FID-based chemosensing systems for amino acids. Using an environment-responsive fluorophore attached to different anchors in order to manipulate the association constants, the fine tuning the displacement process is possible to alter sensing parameters (selectivity, sensitivity). This was demonstrated by supramolecular systems containing 4-amino1,8-naphthalimide fluorophores with three different anchors as guest, and WP5 as host, the first pillararene-based FID systems to detect basic amino acids. These systems also proved applicable for the detection of biogenic diamines.

\section{Results and discussion}

\section{Structures and syntheses}

As the fluorophore unit of the guest molecules, the 4-amino-1,8naphthalimide group was selected, which has the special advantages of convenient derivatization and the easy modulation of its fluorescence via photoinduced electron transfer (PET) process. $^{56}$ In water, the fluorescence of this fluorophore is quenched due to the stabilization of its ICT state. However, when the substituent attached to the 4-position becomes positively charged, the fluorescence is recovered due to the inhibition of the PET process. This principle is widely used for the fluorescent detection of metal ions. ${ }^{57-59}$ Since WPns form strong complexes with several cationic guests, such as ammonium, imidazolium and pyridinium compounds, we speculated that some of these groups would be ideal as anchoring moieties.

The expected sensing mechanism of our FID systems is illustrated in Scheme 1. The complexation of the naphthalimide derivatives by WP5 leads to a fluorescence quenching, since the negative charges on the rim of the pillararene host compensate the positive charge on the anchors of the guests, restoring the PET effect. These systems will then provide a turn-on fluorescence response to the addition of non-fluorescent analytes as competitive guests.

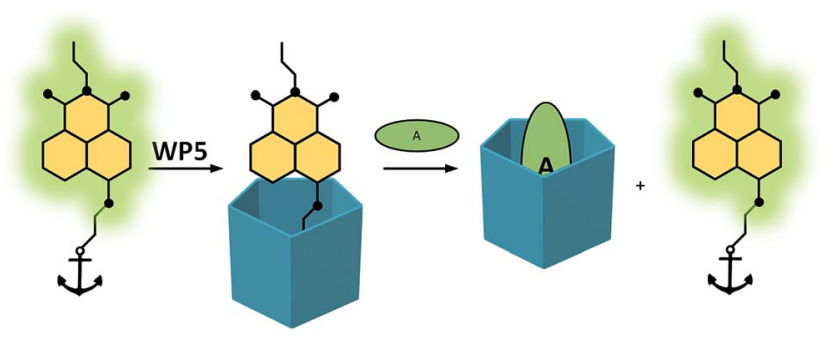

Scheme 1 Anchored naphthalimides in WP5-based indicator displacement systems. The green oval represents the analyte.<smiles>CCCN1C(=O)c2cccc3c(NCCCCN)ccc(c23)C(=O)N1CCC</smiles>

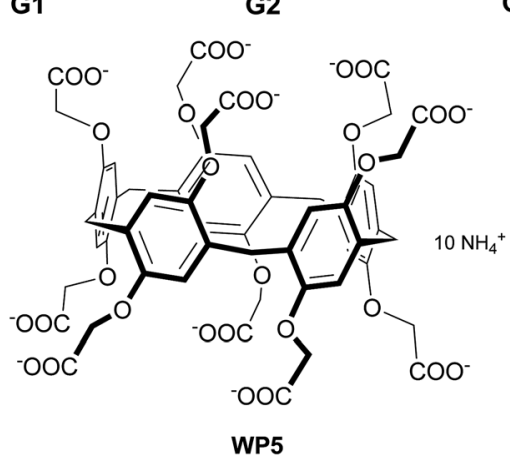

Fig. 1 Structures of the guests G1-G3 and WP5.

Guests G1-G3 (Fig. 1) were synthesized using the general methods to yield substituted naphthalimides (see ESI for synthetic details $\dagger$ ). WP5 was synthesized as described recently. ${ }^{\mathbf{6 0}}$ G1 has a putrescine anchor and is already mentioned in the literature as an anchored indicator for cucurbituril hosts. ${ }^{\mathbf{6 1}} \mathbf{G 2}$ and G3 are new naphthalimide derivatives. G2 contains an imidazolium moiety as anchor and was prepared from 4-(2bromoethylamino)-1,8-naphthalimide ${ }^{57}$ and $N$-methylimidazole. G3 bearing a trimethyl-ammonium end group was synthesized in a similar manner. This latter dye was designed to be a "poorly" complexing indicator since the size of the WP5 cavity allows only a weaker binding of this guest. The compounds were fully characterised using NMR and HRMS techniques. The complexation studies were performed using optical spectroscopic methods and ${ }^{1} \mathrm{H}$-NMR measurements.

\section{Complexation studies}

As had been expected, all the three guests fluoresced well in HEPES-buffered aqueous media ( $\mathrm{pH} 7.4)$ due to the inhibition of the PET process by the primary (G1) and quaternary (G2 and G3) ammonium anchor groups. The results with the spectral properties are summarized in Table 1 . The complexation with the anionic WP5 caused a significant reduction of the fluorescence due to the charge-compensating effects of the carboxylate arms of the host (Fig. 2). Since the positive charge was effectively shielded, the fluorescence decreased due to the increased PET.

The Job's plots of the indicator-WP5 systems (ESI Fig. S12$\mathrm{S} 14 \dagger$ ) indicated a stepwise supramolecular reaction: besides the expected 1:1 complexes, 2:1 (guest:WP5) complexes were also formed. The stepwise association constants for the two 
Table 1 Spectroscopic data of the naphthalimide guests and their WP5 complexes

\begin{tabular}{lllll}
\hline & $\lambda_{\text {abs }}(\mathrm{nm})$ & $\varepsilon\left(\mathbf{M}^{-1} \mathrm{~cm}^{-1}\right)$ & $\lambda_{\mathrm{em}}(\mathrm{nm})$ & $\Phi$ \\
\hline G1 & 452 & 14800 & 550 & 0.12 \\
G1·WP5 & 452 & 17600 & 550 & 0.02 \\
G2 & 438 & 18800 & 535 & 0.20 \\
G2·WP5 & 437 & 14300 & 535 & 0.02 \\
G3 & 434 & 14900 & 533 & 0.32 \\
G3·WP5 & 434 & 14800 & 533 & 0.06
\end{tabular}

complexes were determined by a least square fitting to the fluorescence spectra of samples with the same indicator and different WP5 concentrations (Table 2, see ESI Section 6 for details $\dagger$ ). As at the higher WP5 equivalents, applied in the displacements assays, the dyes were present predominantly in the form of their 1:1 WP5 complexes, the concentrations of $2: 1$ complexes were negligible, we discuss here briefly only the trends in the $K_{\mathrm{a} 1}$ association constants.

The guests showed the expected variations in complex strength, with $K_{\mathrm{a} 1}$ values ranging from $10^{3}$ to $10^{6} \mathrm{M}^{-1}$. These results indicate that an efficient supramolecular control could be achieved by the proper selection of the anchoring groups. In the case of G1, a strong 1:1 complex was formed $\left(K_{\mathrm{a} 1, \mathrm{G} 1}=\right.$ $1.24 \times 10^{6} \mathrm{M}^{-1}$ ) with $\mathrm{WP5}$, resulting in an 8-fold reduction of the fluorescence intensity. G2 also formed an 1:1 complex, with a somewhat lower association constant $\left(K_{\mathrm{a} 1, \mathrm{G} 2}=2.83 \times 10^{5}\right.$ $\mathrm{M}^{-1}$ ) and a similar response in fluorescence. The lowest association constant $\left(K_{\mathrm{a} 1, \mathrm{G} 3}=4.75 \times 10^{3} \mathrm{M}^{-1}\right)$ was measured in the case of G3. This demonstrates that the trimethylammonium group functions as a "poorly complexing" anchor.

The supramolecular interactions in the complexes were further evaluated using ${ }^{1} \mathrm{H}-\mathrm{NMR}$ measurements in $\mathrm{D}_{2} \mathrm{O}$ (Fig. 3). The complexation was fast enough to present one set of signals in each case. In the case of $\mathbf{G 1}$, the protons of the anchor moiety displayed unusually large upfield shifts (up to $\Delta \delta=-3.4 \mathrm{ppm}$ ) making some of the methylene protons appear at negative chemical shifts. The aromatic protons of the fluorophore showed slight downfield shifts with the exception in the 3position near the anchor unit that exhibited some upfield shift.
Table 2 Association constants of the complexes

\begin{tabular}{lll}
\hline & $\log K_{\mathrm{a} 1}\left(K_{\mathrm{a} 1}\right.$ in $\left.\mathrm{M}^{-1}\right)$ & $\log K_{\mathrm{a} 2}\left(K_{\mathrm{a} 2}\right.$ in $\left.\mathrm{M}^{-1}\right)$ \\
\hline G1·WP5 & 6.09 & 6.00 \\
G2·WP5 & 5.45 & 5.75 \\
G3·WP5 & 3.68 & - \\
\hline
\end{tabular}

This can be rationalized by the partial inclusion of the molecule illustrated in Scheme 1: the anchoring unit penetrates the cavity, whereas the naphthalimide moiety remains at the rim of the macrocycle. The protons of the host remained mostly unchanged, which indicates that there was no significant restriction in the movement of the constituent units. Similar effects were observed with G2: large upfield shifts of the $\mathrm{N}$ methylimidazolium protons (up to $\Delta \delta=-1.1 \mathrm{ppm}$ ) and the spacer methylene units (up to $\Delta \delta=-2.4 \mathrm{ppm}$ ) accompanied by the broadening of the signals, which is attributable to the shielding effect of the macrocycle. The aromatic protons of G2 exhibited some downfield shifts as well. Due to the positive charge on the G2 guest, some changes could be detected in the signals of the WP5 macrocyclic host. The sterically less favorable guest G3 showed somewhat different changes: we suppose that due to steric hindering, complete inclusion could not be reached, therefore only small changes were detected in the signals of the anchor moiety (i.e. the trimethylammonium group showed $\Delta \delta=-0.13 \mathrm{ppm})$. The different complexation was also suggested by the upfield shifts of the aromatic protons of the naphthalimide unit.

To gain further insight into these distinct host-guest interactions, we calculated the anchor volumes and compared them to the cavity volume of pillar[5]arene. It was confirmed that the ethylammonium ion (anchor for G1) with a volume of $77 \AA^{3}$, and the $N, N^{\prime}$-ethyl-methyl imidazolium cation (anchor for G2) filling a volume of $143 \AA^{3}$ can be included in the $152 \AA^{3}$ cavity of the WP5 pillarene host, ${ }^{9}$ whereas the volume of $N, N$-ethyltrimethylammonium moiety (anchor for G3) is $171 \AA^{3}$, exceeds the above cavity volume of WP5, preventing such type hostguest interaction (see ESI Section 7 for detailsi).
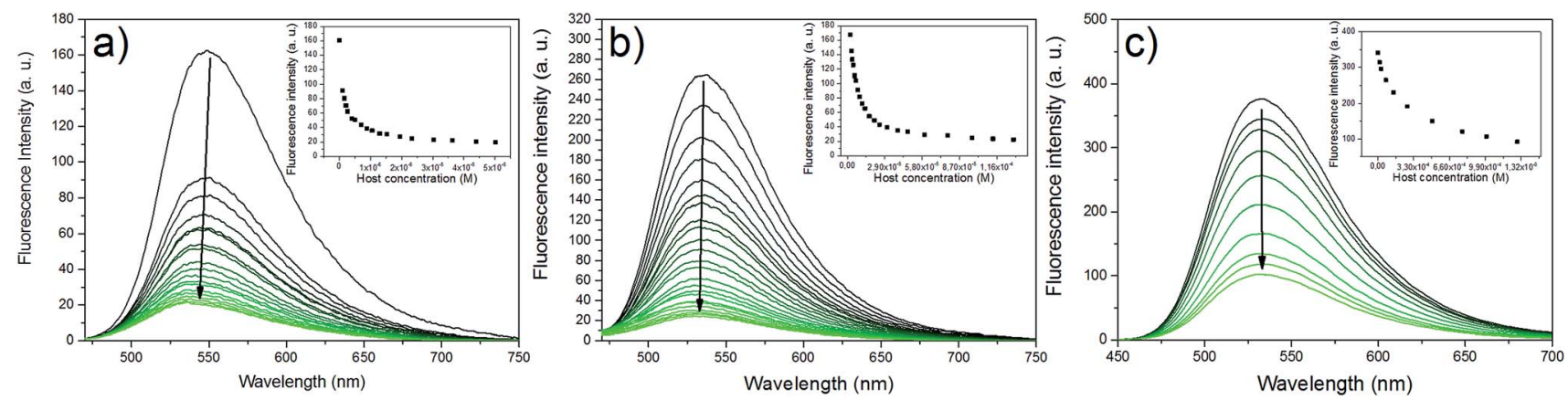

Fig. 2 Fluorescence titration of the guests with WP5 in HEPES buffer (pH 7.4). In each case, the concentration of the indicators was $1 \mu M$. (a) G1 + WP5 ( 0 to 50 equivalent), excitation at $450 \mathrm{~nm}$; (b) G2 + WP5 (0 to 128 equivalent), excitation at $399 \mathrm{~nm}$; (c) G3 + WP5 (0 to 1280 equivalent), excitation at $433 \mathrm{~nm}$; the insets show the fluorescence emission vs. host concentration at $550 \mathrm{~nm}$. 

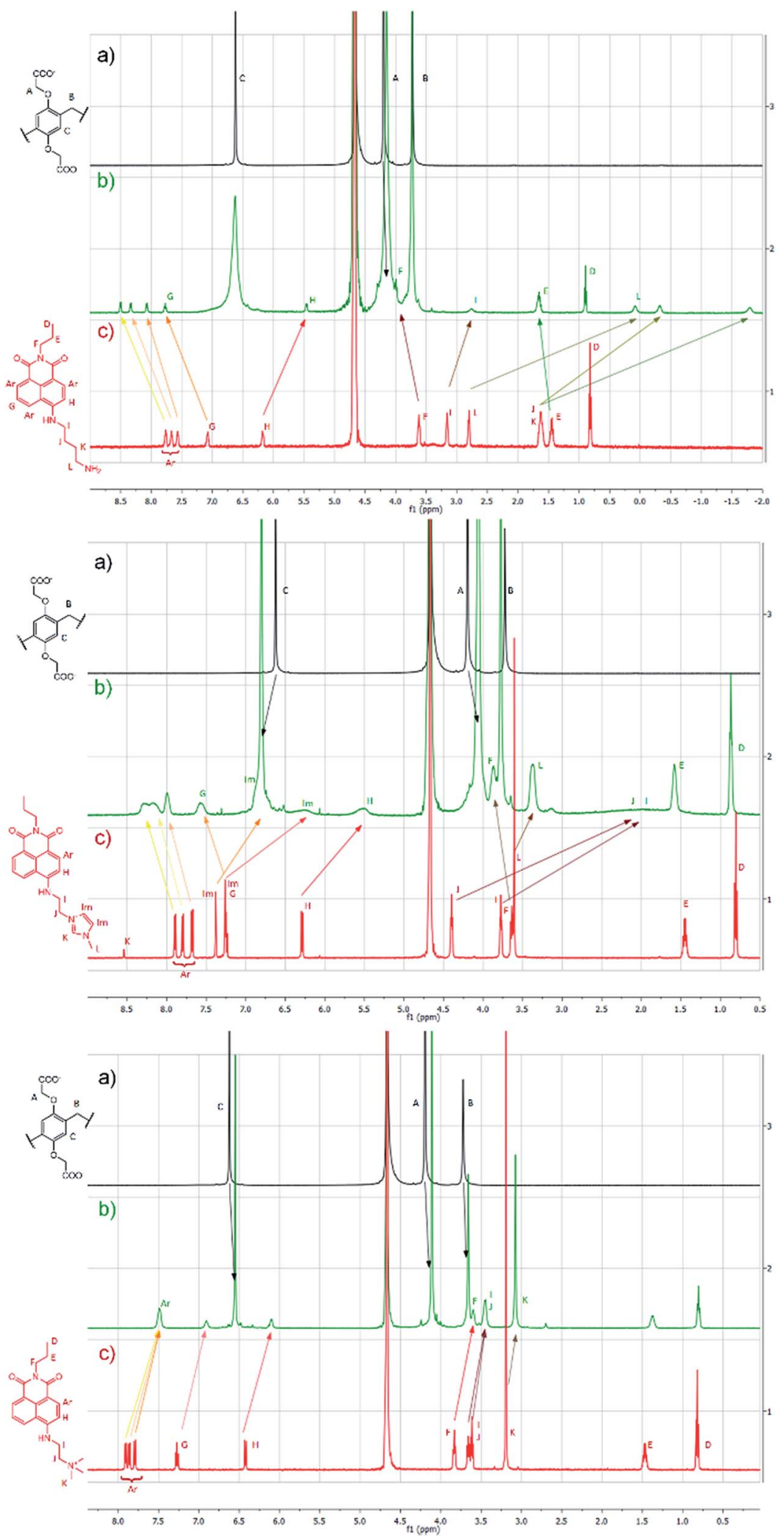

Fig. $3{ }^{1} \mathrm{H}$-NMR spectra $\left(500 \mathrm{MHz}, 25^{\circ} \mathrm{C}\right.$ ) of the guests G1-G3 (a) WP5 (b) Gx.WP5 (c) Gx in $\mathrm{D}_{2} \mathrm{O}$ maintaining a constant concentration of 3 mM for all components.

\section{Fluorescence indicator displacement}

Due to their large signal modulation upon complexation and optimal binding constants with WP5, G1 and G2 were selected as potential indicators for FID systems. Upon addition of various amino acids ( $3 \mathrm{mM})$ to the indicator-WP5 complexes, a significant fluorescence enhancement was observed only in 

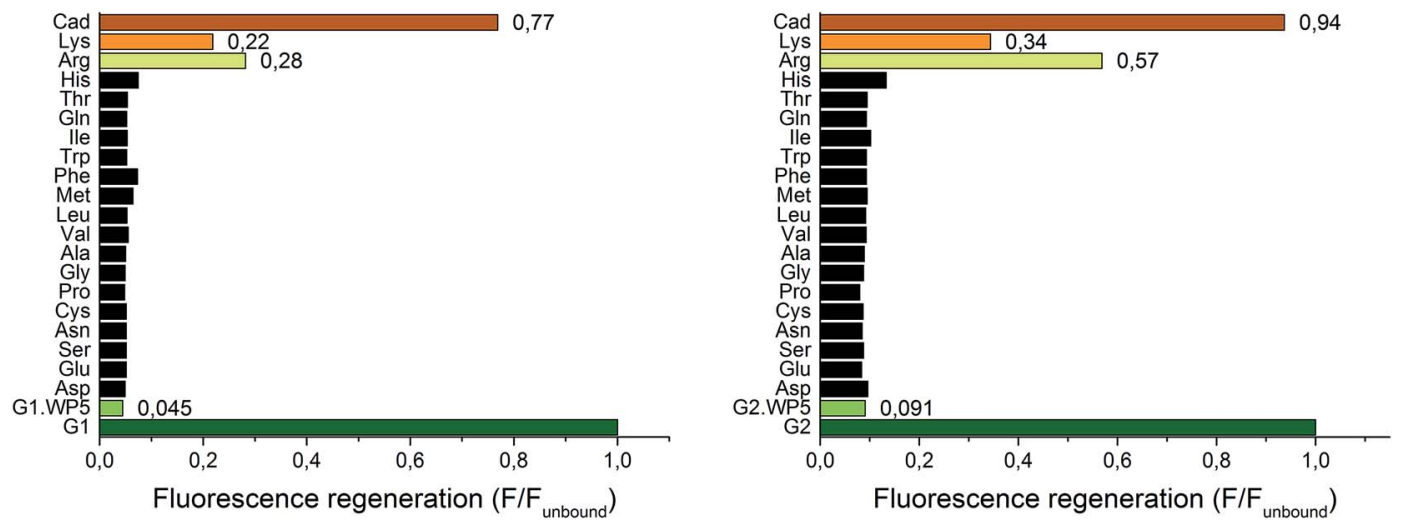

Fig. 4 Fluorescence regeneration values ( $\left.F / F_{\text {unbound }}\right)$ at $550 \mathrm{~nm}$ of cadaverine (Cad) and various amino acids using G1 · WP5 (left, excitation at 450 $\mathrm{nm}$ ) and G2 -WP5 (right, excitation at $399 \mathrm{~nm}$ ) systems as indicator displacement assays. For the screening experiments, the concentrations of the indicators were $3 \mu \mathrm{M}$ using 15 equivalent host. The analytes were added in $3 \mathrm{mM}$ concentration.

the cases of arginine and lysine. The effect could be characterized by the fluorescence regeneration, $F / F_{\text {unbound }}$, which is related to the proportion of the displaced indicators (Fig. 4).

The screening experiment showed that the addition of nonbasic $\alpha$-amino acids in high concentration caused negligible changes in the fluorescence, with the exception of histidine, which resulted in a small fluorescence enhancement. Thus, both of our FID systems showed a high selectivity towards the basic amino-acids. The two diamines, putrescine and cadaverine caused an almost complete fluorescence regeneration of the free indicators (only cadaverine shown). Subsequently, titration experiments were performed to evaluate the sensitivity of the two indicator displacement systems for cadaverine, arginine and lysine (Fig. S15 and S16 $\dagger$ ). A host concentration of $15 \mu \mathrm{M}$ was selected (15-fold excess related to the indicator guest) to achieve a high level of indicator-WP5 complex, which was $\sim 95 \%$ for the G1 $\cdot$ WP5 and $\sim 80 \%$ for the G2 $\cdot$ WP5 system as can be calculated from the respective $K_{\mathrm{a}}$ values.

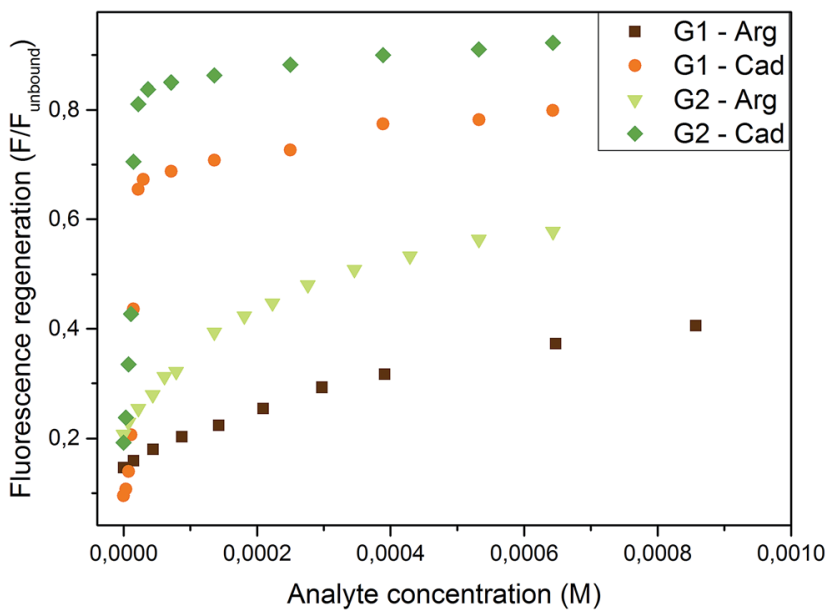

Fig. 5 Fluorescence regeneration ( $\left.F / F_{\text {unbound }}\right)$ of indicators G1 and G2 at $550 \mathrm{~nm}$, caused by their displacements from their WP5 complexes by arginine and cadaverine. In this case, $1 \mu \mathrm{M}$ indicator concentration and 15 equivalent of host were used.
Both systems were found highly sensitive for cadaverine with a large turn-on fluorescence. A smaller, but still significant enhancement was induced by arginine and an even smaller enhancement was reached with lysine. The weaker G2 WP5 complex showed the expected higher sensitivity towards the analytes, as can be seen in Fig. 5 . The limit of detection (LOD; for its calculation see ESI Section $8 \dagger$ ) was found to be larger by almost one order of magnitude in the case of G2.WP5: for cadaverine, the LOD was found to be $9.44 \times 10^{-7} \mathrm{M}$ and $1.41 \times$ $10^{-7} \mathrm{M}$ for G1 $\cdot \mathbf{W P 5}$ and $\mathbf{G 2} \cdot \mathbf{W P 5}$, respectively. In the case of arginine, the LOD value of $1.34 \times 10^{-5} \mathrm{M}$ was obtained for the G1 WP5 assay and $3.27 \times 10^{-6} \mathbf{M}$ for the G2 $\cdot$ WP5 assay. This example demonstrates that the sensing properties can be tailored and fine-tuned with varying the anchor unit with the same fluorophore. In addition, due to the different sensitivity of these systems, differential sensing can be achieved based on the anchored naphthalimide indicators and WP5.

The indicator displacement was further evaluated by ${ }^{1} \mathrm{H}-$ NMR-spectroscopy. Following the NMR investigation of the naphthalimide-WP5 mixtures (see Section 'Complexation studies'), the mixtures of the guests with cadaverine and arginine were tested in the absence of the host (Fig S17-S20†). No significant changes were observed showing the lack of interaction of the indicators with the analytes. In the case of G1, the slight shift of the signals could be attributed to the $\mathrm{pH}$ sensitivity of the anchor group. Next, the indicator displacement process was studied by NMR spectroscopy (Fig. S21-S24†). Upon addition of cadaverine in 10-fold excess to the complexes of WP5 with G1 and G2, the displacement of the indicators was clearly visible, as the spectra of the free indicators were regained. However, when arginine was added to the G1 $\cdot$ WP5 complex in the same excess, an average signal of the complex and the free $\mathbf{G} \mathbf{2}$ was observed as a result of partial displacement. Unfortunately, upon the addition of arginine to G1.WP5 precipitation occurred, it is unlikely, however, that in the latter system the indicator was displaced from the strong complex.

With respect to the acid-base character of components, some aspects of the $\mathrm{pH}$-dependence of the complexation and displacement reactions were also investigated. As could be 
expected, the complexation of G1 was pH-dependent (ESI Section 10†), however, the association constants for G2 WP5 were almost identical in the $6.8-8.2 \mathrm{pH}$ range. The indicator displacement of G2 $\cdot$ WP5 was tested at $\mathrm{pH}$ values $6.8,7.4$ and 8.2: evidently, higher fluorescence regeneration values were detected for the basic amino acids at lower $\mathrm{pH}$, whereas, for cadaverine, no correlation was found (Fig. S25 $\dagger$ ).

As a further test of the G2 -WP5 system, the more sensitive FID sensor for basic amino acids, the association constants for the lysine-WP5 and arginine-WP5 supramolecular complexes were determined by a least square fitting to the fluorescence spectra of G2-WP5-lysine/arginine mixtures, with different lysine/arginine concentrations. For the lysine-WP5 complex $K_{\mathrm{a}}=1.12 \times 10^{3} \mathrm{M}^{-1}$ was obtained, for the arginine-WP5 complex $K_{\mathrm{a}}=5.24 \times 10^{3} \mathrm{M}^{-1}$, in good agreement with the values of $1.8 \times 10^{3} \mathrm{M}^{-1}$ for lysine-WP5 and $5.9 \times 10^{3} \mathrm{M}^{-1}$ for arginine-WP5 determined by NMR experiments. ${ }^{28}$

\section{Conclusions}

In conclusion, the synthesized 4-amino-1,8-naphthalimide indicators having different anchoring groups in the 4-position were shown to form complexes with WP5 with varying association constants and large signal modulation. Two complexes were tested as indicator displacement systems for cadaverine, putrescine and $\alpha$-amino acids. Both systems can detect cadaverine and the basic amino acids with high selectivity and different sensitivity. The FID process was further established using NMR spectroscopy. To our knowledge, this is the first WP5-based FID sensing system for analytes with biological relevance. We believe that the current toolbox of indicator displacement assays could be further expanded with this intriguing host.

\section{Acknowledgements}

We thank the Hungarian Research Foundation for the financial support of this work (Grant No. K108752). We are grateful to Fanni Bazsó for the mass spectrometric measurements and Zoltán Szakács for the anchor volume calculations.

\section{Notes and references}

1 E. V. Anslyn, J. Org. Chem., 2007, 72, 687-699.

2 L. You, D. Zha and E. V. Anslyn, Chem. Rev., 2015, 115, 78407892.

3 B. T. Nguyen and E. V. Anslyn, Coord. Chem. Rev., 2006, 250, 3118-3127.

4 R. N. Dsouza, U. Pischel and W. M. Nau, Chem. Rev., 2011, 111, 7941-7980.

5 G. Ghale and W. M. Nau, Acc. Chem. Res., 2014, 47, 21502159.

6 T. Ogoshi, S. Kanai, S. Fujinami, T.-a. Yamagishi and Y. Nakamoto, J. Am. Chem. Soc., 2008, 130, 5022-5023.

7 P. J. Cragg and K. Sharma, Chem. Soc. Rev., 2012, 41, 597-607. 8 T. Ogoshi, J. Inclusion Phenom. Macrocyclic Chem., 2012, 72, 247-262.
9 M. Xue, Y. Yang, X. Chi, Z. Zhang and F. Huang, Acc. Chem. Res., 2012, 45, 1294-1308.

10 T. Ogoshi and T.-a. Yamagishi, Eur. J. Org. Chem., 2013, 2013, 2961-2975.

11 H. Zhang and Y. Zhao, Chem.-Eur. J., 2013, 19, 16862-16879.

12 D. Cao and H. Meier, Asian J. Org. Chem., 2014, 3, 244-262.

13 C. Li, Chem. Commun., 2014, 50, 12420-12433.

14 D. Cao and H. Meier, Synthesis, 2015, 26, 1041-1056.

15 K. Yang, Y. Pei, J. Wen and Z. Pei, Chem. Commun., 2016, 52, 9316-9326.

16 T. Ogoshi, T. A. Yamagishi and Y. Nakamoto, Chem. Rev., 2016, 116, 7937-8002.

17 Y. Wang, G. Ping and C. Li, Chem. Commun., 2016, 52, 98589872.

18 T. Ogoshi, M. Hashizume, T.-a. Yamagishi and Y. Nakamoto, Chem. Commun., 2010, 46, 3708-3710.

19 G. Yu, M. Xue, Z. Zhang, J. Li, C. Han and F. Huang, J. Am. Chem. Soc., 2012, 134, 13248-13251.

20 Y. Ma, X. Ji, F. Xiang, X. Chi, C. Han, J. He, Z. Abliz, W. Chen and F. Huang, Chem. Commun., 2011, 47, 12340-12342.

21 G. C. Yu, J. Zhou, J. Shen, G. P. Tang and F. H. Huang, Chem. Sci., 2016, 7, 4073-4078.

22 Y. Yao, J. Li, J. Dai, X. Chi and M. Xue, RSC Adv., 2014, 4, 9039-9043.

23 W. Cheng, H. Tang, R. Wang, L. Wang, H. Meier and D. Cao, Chem. Commun., 2016, 52, 8075-8078.

24 C. Li, X. Shu, J. Li, S. Chen, K. Han, M. Xu, B. Hu, Y. Yu and X. Jia, J. Org. Chem., 2011, 76, 8458-8465.

25 S. Dasgupta, A. Chowdhury and P. S. Mukherjee, RSC Adv., 2015, 5, 85791-85798.

26 B. Shi, K. Jie, Y. Zhou, J. Zhou, D. Xia and F. Huang, J. Am. Chem. Soc., 2016, 138, 80-83.

27 Y. Sun, F. Guo, T. Zuo, J. Hua and G. Diao, Nat. Commun., 2016, 7, 12042.

28 C. Li, J. Ma, L. Zhao, Y. Zhang, Y. Yu, X. Shu, J. Li and X. Jia, Chem. Commun., 2013, 49, 1924-1926.

29 N. L. Strutt, R. S. Forgan, J. M. Spruell, Y. Y. Botros and J. F. Stoddart, J. Am. Chem. Soc., 2011, 133, 5668-5671.

30 P. Wang, X. Yan and F. Huang, Chem. Commun., 2014, 50, 5017-5019.

31 P. Wang, Y. Yao and M. Xue, Chem. Commun., 2014, 50, 5064-5067.

32 M. Bojtar, Z. Szakacs, D. Hessz, M. Kubinyi and I. Bitter, $R S C$ Adv., 2015, 5, 26504-26508.

33 M. Bojtár, Z. Szakács, D. Hessz, F. L. Bazsó, M. Kállay, M. Kubinyi and I. Bitter, Dyes Pigm., 2016, 133, 415-423.

34 J. Le Boucher, C. Charret, C. Coudray-Lucas, J. Giboudeau and L. Cynober, Clin. Chem., 1997, 43, 1421-1428.

35 G. L. Luque, N. F. Ferreyra and G. A. Rivas, Talanta, 2007, 71, 1282-1287.

36 Y. Zhou and J. Yoon, Chem. Soc. Rev., 2012, 41, 52-67.

37 A. Buryak and K. Severin, J. Am. Chem. Soc., 2005, 127, 37003701.

38 X. Zhou, X. Jin, D. Li and X. Wu, Chem. Commun., 2011, 47, 3921-3923.

39 L. He, V. L. L. So and J. H. Xin, Sens. Actuators, B, 2014, 192, 496-502. 
40 J. Cao, L. Ding, Y. Zhang, S. Wang and Y. Fang, J. Photochem. Photobiol., A, 2016, 314, 66-74.

41 D. Xiong, M. Chen and H. Li, Chem. Commun., 2008, 880882, DOI: $10.1039 /$ B716270G.

42 G. Patel and S. Menon, Chem. Commun., 2009, 3563-3565, DOI: $10.1039 /$ B905141D.

43 K. A. Rawat and S. K. Kailasa, Microchim. Acta, 2014, 181, 1917-1929.

44 D. M. Bailey, A. Hennig, V. D. Uzunova and W. M. Nau, Chem.-Eur. J., 2008, 14, 6069-6077.

45 L. A. Baumes, M. Buaki, J. Jolly, A. Corma and H. Garcia, Tetrahedron Lett., 2011, 52, 1418-1421.

46 T. Minami, N. A. Esipenko, B. Zhang, L. Isaacs and P. Anzenbacher, Chem. Commun., 2014, 50, 61-63.

47 X. Lu, W. Wang, Q. Dong, X. Bao, X. Lin, W. Zhang, X. Dong and W. Zhao, Chem. Commun., 2015, 51, 1498-1501.

48 X. Qian, W. Gong, F. Wang, Y. Lin and G. Ning, Tetrahedron Lett., 2015, 56, 2764-2767.

49 K. Secor, J. Plante, C. Avetta and T. Glass, J. Mater. Chem., 2005, 15, 4073-4077.

50 G. Lu, J. E. Grossman and J. B. Lambert, J. Org. Chem., 2006, 71, 1769-1776.

51 S. Reinert and G. J. Mohr, Chem. Commun., 2008, 2272-2274, DOI: $10.1039 / B 717796 H$.
52 M.-S. Steiner, R. J. Meier, A. Duerkop and O. S. Wolfbeis, Anal. Chem., 2010, 82, 8402-8405.

53 B. Lee, R. Scopelliti and K. Severin, Chem. Commun., 2011, 47, 9639-9641.

54 J.-F. Lin, J. Kukkola, T. Sipola, D. Raut, A. Samikannu, J.-P. Mikkola, M. Mohl, G. Toth, W.-F. Su, T. Laurila and K. Kordas, J. Mater. Chem. A, 2015, 3, 4687-4694.

55 Y. Hu, X. Ma, Y. Zhang, Y. Che and J. Zhao, ACS Sens., 2016, 1, 22-25.

56 R. M. Duke, E. B. Veale, F. M. Pfeffer, P. E. Kruger and T. Gunnlaugsson, Chem. Soc. Rev., 2010, 39, 3936-3953.

57 T. Chen, W. Zhu, Y. Xu, S. Zhang, X. Zhang and X. Qian, Dalton Trans., 2010, 1316-1320.

58 G. R. C. Hamilton, S. K. Sahoo, S. Kamila, N. Singh, N. Kaur, B. W. Hyland and J. F. Callan, Chem. Soc. Rev., 2015, 44, 4415-4432.

59 J. Yin, Y. Hu and J. Yoon, Chem. Soc. Rev., 2015, 44, 46194644.

60 R. R. Kothur, J. Hall, B. A. Patel, C. L. Leong, M. G. Boutelle and P. J. Cragg, Chem. Commun., 2014, 50, 852-854.

61 C. P. Carvalho, R. Ferreira, J. P. Da Silva and U. Pischel, Supramol. Chem., 2013, 25, 92-100. 